\title{
Prevalence, Diagnosis, Perioperative Monitoring and Treatment of Right Ventricular Dysfunction and/or Pulmonary Arterial Hypertension in Cardiac Surgical Patients in Germany-A Postal Survey
}

\author{
${ }^{1}$ Department of Anesthesiology and Intensive Care, University of \\ Lübeck, Lübeck, Germany \\ 2 Department of Cardiovascular Surgery, Christian-Albrechts \\ University, Kiel, Germany \\ ${ }^{3}$ Department of Anesthesiology and Intensive Care, Charité University \\ Hospital, Charité Campus Mitte and Virchow Klinikum, Berlin, Germany \\ ${ }^{4}$ Department of Cardiovascular Surgery, German Armed Forces \\ Central Hospital, Koblenz, Germany \\ ${ }^{5}$ Department of Anesthesiology, University Hospital Hamburg \\ Eppendorf, Hamburg, Germany \\ ${ }^{6}$ Department of Anesthesiology and Intensive Care, Saarland \\ University Medical Center, Homburg/Saar, Germany \\ 7 Department of Cardiovascular and Vascular Surgery, Heart Center \\ University Freiburg, Freiburg, Germany \\ 8 Department of Thoracic and Cardiovascular Surgery, University \\ Hospital Essen, Essen, Germany \\ ${ }^{9}$ Department of Anesthesiology and Intensive Care Medicine, St. \\ Georg Hospital, Hamburg, Germany \\ 10 Department of Anesthesiology, Heart and Diabetic Center NRW, \\ Ruhr University of Bochum, Bad Oeynhausen, Germany
}

Matthias Heringlake ${ }^{1}$ Julika Schön ${ }^{1}$ Teresa Pliet ${ }^{1}$ Nils Haake ${ }^{2}$ Alexander Reinecke ${ }^{2}$ Marit Habicher ${ }^{3}$ Michael Sander ${ }^{3}$ Andreas Markewitz ${ }^{4}$ Daniel A. Reuter ${ }^{5}$ Heinrich Volker Groesdonk ${ }^{6}$ Georg Trummer ${ }^{7}$ Kevin Pilarzyk ${ }^{8}$ Michael von der Brelie ${ }^{2}$ Berthold Bein ${ }^{9} \quad$ Uwe Schirmer $^{10}$

Address for correspondence Prof. Dr. Matthias Heringlake, Department of Anesthesiology and Intensive Care, University of Lübeck 23538 Lübeck, Germany (e-mail: matthias.heringlake@uksh.de).

Thorac Cardiovasc Surg 2017;65:593-600.

\author{
Abstract \\ Keywords \\ - cardiac \\ - pulmonary vascular \\ resistance/ \\ hypertension \\ - surgery \\ - complications
}

Background Sparse data are available on the prevalence of right ventricular dysfunction and/or pulmonary arterial hypertension in patients scheduled for cardiac surgery in Germany as well as on the intensity and modalities used for diagnosis, perioperative monitoring, and treatment of these comorbidities.

Methods A postal survey including questions on the prevalence of preoperative right ventricular dysfunction and/or pulmonary arterial hypertension in patients undergoing cardiac surgery in 2009 was sent to 81 German heart centers. Total 47 of 81 (58\%) heart centers returned the questionnaires. The centers reported data on 51,095 patients, and $49.8 \%$ of the procedures were isolated coronary artery bypass grafting.

Results Data on the prevalence of preoperative pulmonary hypertension and/or right ventricular dysfunction were not available in $54 \%$ and $64.6 \%$ of centers. In the remaining hospitals, $19.5 \%$ of patients presented right heart dysfunction and $10 \%$ pulmonary received

November 1, 2015

accepted after revision

January 12, 2016

published online

February 24, 2016 (c) 2017 Georg Thieme Verlag KG

Stuttgart · New York
DOI https://doi.org/

10.1055/s-0036-1572511. ISSN 0171-6425. 
arterial hypertension. Preoperative echocardiography was performed in only $45.3 \%$ of the coronary artery bypass grafting cases. Preoperative pharmacologic treatment of pulmonary hypertension or right ventricular dysfunction with oral sildenafil, inhaled prostanoids, or nitric oxide was initiated in $71 \%$ and $95.7 \%$ of the centers, respectively. Intra- and postoperative treatment was most frequently accomplished with phosphodiesterase-III inhibitors.

Conclusion The prevalence of preoperative right heart dysfunction and pulmonary arterial hypertension in cardiac surgical patients in Germany seems to be substantial. However, in more than $50 \%$ of the patients, no preoperative data on right ventricular function and pulmonary arterial pressure are available. This may lead to underestimation of perioperative risk and inappropriate management of this high-risk population.

\section{Introduction}

It has become increasingly clear within the last years that right ventricular dysfunction (RVD) is a rather frequent complication after cardiac surgery, is more difficult to treat than left ventricular dysfunction, and may negatively impact patient survival with mortality rates up to $75 \%{ }^{1}$ Despite a rather low $(0.1 \%)$ incidence of overt RVD, following uncomplicated cardiac surgical procedures, 2 to $3 \%$ of patients after heart transplantation and 20 to $30 \%$ of patients receiving a left ventricular assist device (LVAD) develop this complication. ${ }^{1,2}$ Furthermore, up to $40 \%$ of patients presenting with cardiogenic shock after cardiac surgery show RVD. ${ }^{3}$

Overt ischemic postoperative RVD may easily be explained as a consequence of severe left-sided heart failure but may also be induced by incomplete revascularization, inadequate cardioplegia, bypass graft malfunction, or, less frequently, due to surgical complications of valve procedures. ${ }^{1,4}$ Additionally, more subtle RVD has been reported to occur almost universally in patients undergoing on- and off-pump CABG surgery and has been shown to be detectable up to 3 months after surgery. ${ }^{5}$ The pathophysiologic mechanisms causing this prolonged RVD after uncomplicated coronary artery bypass grafting (CABG) surgery are still a matter of debate. However, one important mechanism seems to be pericardiotomy itself. Animal data point to a disturbed right ventricular myocardial blood flow after pericardiotomy. ${ }^{6}$ In line with this, Unsworth and colleagues have shown a loss in right ventricular contractility immediately after pericardial incision. ${ }^{7}$

It is well accepted that right heart dysfunction is a poor prognostic sign in patients with heart failure ${ }^{8,9}$ and that up to $50 \%$ of heart failure patients with preserved left ventricular ejection fraction present RVD. ${ }^{10}$ In contrast to the course of chronic heart failure, little is known about the effects of preoperative RVD on outcome after cardiac surgery. Recently, Garatti and coworkers analyzed the impact of preoperative RVD on perioperative complications and long-term outcome in 324 patients with ischemic heart failure undergoing surgical ventricular reconstruction. Twenty-one percent of patients presented preoperative RVD and had a significantly complicated perioperative course; that is, they had a higher incidence of low-cardiac output syndrome and need for intra-aortic counterpulsation. Multivariate analysis identified this variable as an independent predictor of late mortality. ${ }^{11}$ No data about the incidence of preoperative RVD in patients scheduled for cardiac surgery in Germany are yet available.

Pulmonary arterial hypertension (PAH) is a relevant risk factor in cardiac surgical patients and has been included in various cardiac surgical risk scores, including the most frequently used score in Germany, the EuroSCORE. ${ }^{12}$ According to the institution in charge of external quality assurance required by law in Germany "AQUA," $2.7 \%$ of patients undergoing $C A B G$ and $11.8 \%$ of patients undergoing combined aortic valve surgery and CABG in 2012 had preoperative PAH (https://www.sqg.de/ergebnisse/leistungsbereiche/index.html; accessed on 21.4.2015; information in German language). Sparse data are available about the prevalence of PAH in patients scheduled for other procedures, that is, mitral valve surgery and on optimal treatment strategies for patients with PAH and/or RVD. ${ }^{13}$

To shed some light on the magnitude of this problem and as a base for future prospective trials, the present study aimed at assessing the incidence of preoperative right ventricular dysfunction/failure and $\mathrm{PAH}$, measures used to diagnose these risk factors, and treatment practice in adult cardiac surgery in Germany by a postal survey.

\section{Material and Methods}

The Section "Hemodynamics" of the German Interdisciplinary Association for Intensive Care and Emergency Medicine and the "Scientific Working group on Cardiac Anesthesia" of the German Society of Anesthesiology and Intensive Care Medicine developed a questionnaire focusing on adult patients undergoing cardiac surgery. This questionnaire was sent to 81 German heart centers in October 2010. The period of interest was the year 2009 .

The questionnaire included questions on general issues regarding size and organization of the center and the perioperative care (Part A), questions concerning preoperative diagnosis including data on the prevalence of RVD and/or PAH (Part B), and questions on postoperative treatment (Part C). Multiple choice or open questions were used. 


\section{Statistical Analysis}

Data were analyzed by descriptive statistics using the MedCalc 15.2.2 statistical software package (MedCalc Software bvba, Mariakerke, Belgium).

\section{Results}

Of 81 centers, 47 (58\%) returned the questionnaire.

\section{Part A: General Questions:}

\section{Patient Population}

Data on 51,095 adult cardiac surgical patients were reported. The number of adult patients treated in the participating centers in the period of interest ranged from 330 to 3,312 patients (mean 1,135 points), of whom 9.5\% were operated off-pump. The proportion of urgent and emergency cases was $15 \% ; 49.8 \%$ of the procedures were isolated CABG of which $64.2 \%$ included a bypass on the right coronary artery (RCA). In addition, $12.9 \%$ were isolated aortic valve replacements, $5.4 \%$ isolated mitral valve reconstruction or replacement, $0.5 \%$ isolated tricuspid valve procedures, $11.1 \%$ combined procedures, and $13.0 \%$ others.

\section{Surgical Course and Anesthesia}

The mean duration of cardiopulmonary bypass (CBP) for isolated CABG procedures was 59 minutes (range 31-90 minutes). The mean aim for perfusion pressure during $\mathrm{CPB}$ was $60 \mathrm{~mm} \mathrm{Hg}$ (range 30-75 $\mathrm{mm} \mathrm{Hg}$ ) and the mean temperature was $34.3^{\circ} \mathrm{C}$ (range $30-37^{\circ} \mathrm{C}$ ). Nearly $60.9 \%$ of the centers use volatile anesthetics during CBP on a regular basis, $4.4 \%$ occasionally, and $34.8 \%$ never.

\section{Organization of Perioperative Care}

Of all the centers, $34.4 \%$ run a postanesthesia care unit (PACU). The immediate postoperative care was in the responsibility of anesthesiologists in $33.3 \%$ of the participating centers, of cardiac surgeons in $26.7 \%$, and in a combination of both in $40.0 \%$ of the hospitals, respectively. The intensive care unit (ICU) for cardiac surgical patients was led by anesthesiologists in $43.5 \%$ of the hospitals, by cardiac surgeons in $39.1 \%$, by internal specialists in $2.2 \%$, and by an interdisciplinary approach in $15.2 \%$.

\section{Part B: Preoperative Diagnostics}

Of all the centers, $74.5 \%$ stated that the answers on preoperative diagnostics given were estimates, and the other centers reported that at least some data had been drawn from the hospital's data quality register or from a patient data management system.

The centers reported that on average $45.3 \%$ (range $0-100 \%$ ) of patients scheduled for CABG had a preoperative echocardiographic examination with evaluation of pulmonary artery pressure (PAP) and right ventricular function. Nearly $47.7 \%$ of these echocardiographic examinations were performed in the hospital before surgery. About $13.6 \%$ of the centers could not detail any information on the frequency of preoperative echocardiographic testing.

The variables most often rated to be particularly useful for estimation of right ventricular function included dimensions of the right atrium and ventricle, PAP, and tricuspid annular plane systolic excursion (TAPSE). The variables mentioned to be useful for right ventricular assessment are shown in - Fig. 1.

Preoperative right heart catheterization was performed in a median of $5 \%(0-100 \%)$ of patients scheduled for CABG and

\section{[n]}

$$
30
$$

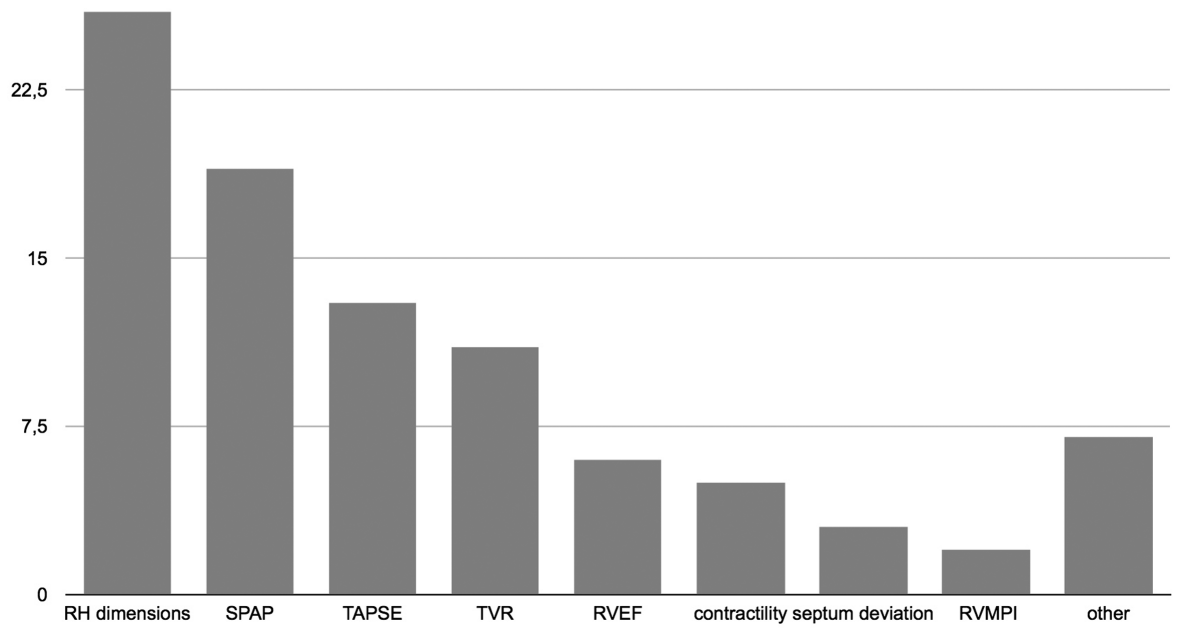

Fig. 1 Variables assessed as especially useful or important for the preoperative echocardiographic assessment of right ventricular function. $n$, number of answers (multiple answers were possible); RH dimensions, right atrial and ventricular diameters; RVEF, right ventricular ejection fraction; RVMPI, right ventricular myocardial performance index; SPAP, systolic pulmonary arterial pressure; TAPSE, tricuspid annular plane systolic excursion; TVR, tricuspid valve regurgitation. 


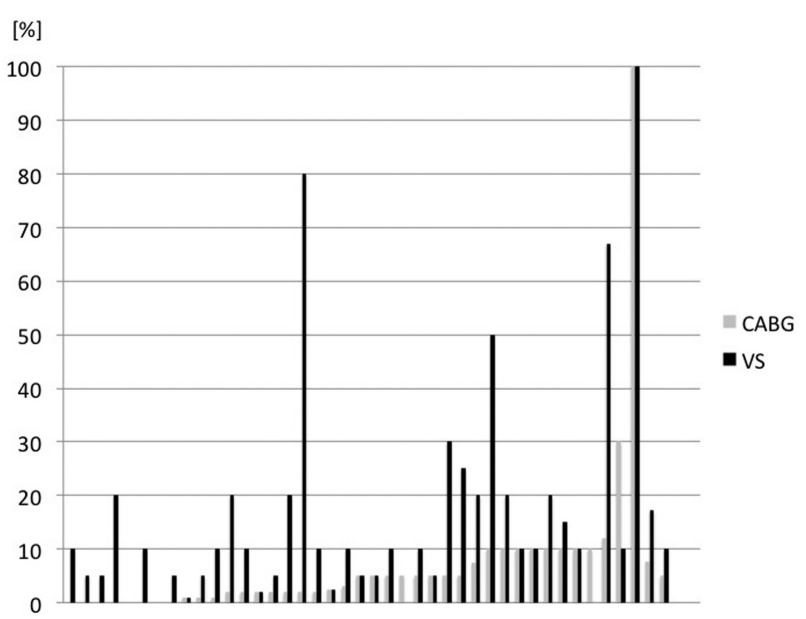

Fig. 2 The frequency of preoperative right heart catheterization in patients scheduled for coronary artery bypass grafting (CABG; gray bars) and valve surgery (VS; black bars).

in a median of $10 \%(0-100 \%)$ of patients planned for valve procedures (-Fig. 2). Nearly $10.3 \%$ of the centers could not report the frequency of preoperative right heart catheterization.

On average, $80 \%$ (2.5-100\%) of patients had preoperative testing of pulmonary function. In addition to echocardiography and pulmonary function testing, only 8 (17.8\%) of the centers performed additional diagnostic tests to evaluate right ventricular function (magnetic resonance imaging [six mentions]; computed tomographic scans [two mentions]; and angiography, scintigraphy, and spiroergometry [one mention each]).

Nearly $72.1 \%$ of the participating centers stated that there are specific groups of cardiac surgical patients in which the risk for RVD is underestimated or even overlooked. - Fig. 3 shows the conditions most frequently thought to be at risk for underestimation of RVD.

In centers in which data on preoperative PAPs were available, a mean incidence of $10 \%(1-100 \%)$ was reported for $\mathrm{PAH}$ (defined as a systolic PAP $>60 \mathrm{~mm} \mathrm{Hg}$ according to the EuroSCORE 1 criteria). The diagnosis was established in $53.8 \%$ by echocardiography and in $24.5 \%$ by right heart catheterization. In the remaining cases, the diagnostic modality could not be specified. Nearly $56.5 \%$ of the centers could not answer how often patients presented with preoperative pulmonary hypertension. Preoperative treatment of patients with severe pulmonary hypertension was reported from $71.1 \%$ of the centers. Oral sildenafil was the most often used drug with $71.1 \%$, oral endothelin antagonists were used by $32 \%$, and intravenous levosimendan was used in $20 \%$ of the centers (more than one answer was possible).

In centers in which data on preoperative right heart function was available, $19.5 \%$ of patients were reported to present with RVD. Of these, 70\% were diagnosed by echocardiography, $15 \%$ by right heart catheterization, and in $15 \%$ the diagnostic modality could not be reported. Again, $66.0 \%$ of centers could not report the preoperative prevalence of patients with RVD. However, 95.7\% of the centers quoted to start therapeutic measures in case of preoperatively diagnosed RVD. The most frequently mentioned drugs included

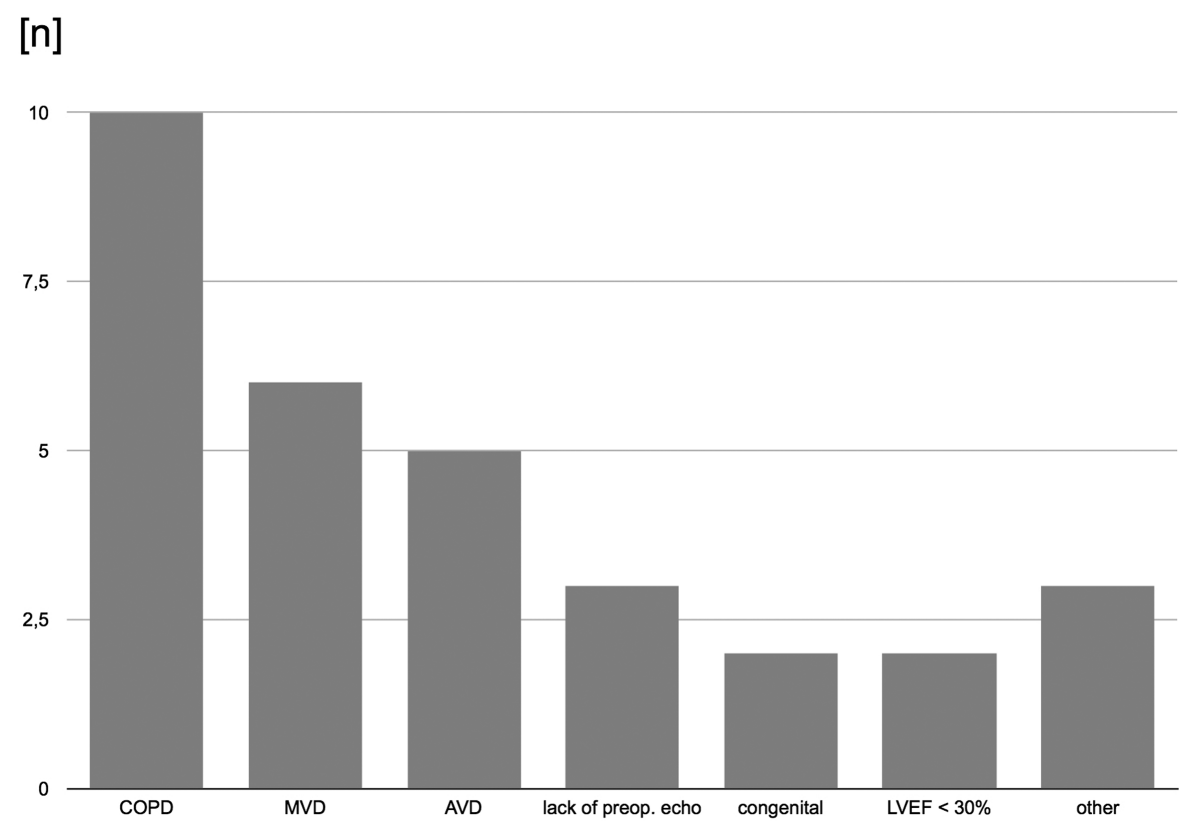

Fig. 3 Cardiac surgical procedures in which the respondents of the survey deemed preoperative right ventricular dysfunction to be frequently underestimated or overlooked. AVD, aortic valve disease; congenital: status after correction of congenital heart disease; COPD, chronic obstructive lung disease; MVD, mitral valve disease; congenital, status after correction of congenital heart disease; $n$, number of answers (multiple answers were possible). 
inhaled prostanoids or nitric oxide (44.2\%), intravenous milrinone (41.9\%), and oral sildenafil (34.9\%).

\section{Part C: Perioperative Management of Patients with Pulmonary Hypertension or Right Ventricular Dysfunction}

Most centers stated that they had standard procedures for monitoring and treatment of RVD or pulmonary hypertension (-Fig. 4). Hemodynamic monitoring for patients with PAH was performed in $87.2 \%$ by use of a pulmonary artery catheter and/or in $66.1 \%$ by transthoracic echocardiography (TEE). Only $25.3 \%$ of the centers used TTE as the first choice for monitoring of patients with pulmonary hypertension (more than one answer was possible).

For patients with RVD, TEE and the pulmonary artery catheter were mentioned equally frequent by $74.5 \%$ of the centers as the first choice for monitoring. TTE was mentioned by $19.2 \%$, and only $6.4 \%$ was quoted to use minimally invasive hemodynamic monitoring-that is, pulse pressure-based methods-for monitoring patients with RVD.

Intra- or postoperative pulmonary hypertension not caused by left ventricular dysfunction was quoted to be primarily treated with intravenous phosphodiesterase-III (PDE-III) blockers (70.2\% of the centers), inhaled prostanoids (38.8\%), intravenous nitroglycerine (31.9\%), and inhaled nitric oxide (25.5\%).

Of all the centers, $68.1 \%$ that they had no information on how many patients needed treatment for postoperative RVD. Those centers having provided this information quoted that 3.2 to $20 \%$ of the patients needed postoperative therapy for right ventricular dysfunction or failure. During a normotensive hemodynamic situation, RVD was most often treated with PDE-III blockers (66.0\%), inhaled prostanoids (36.2\%), intravenous nitroglycerine (29.8\%), inhaled nitric oxide
(25.5\%), and dobutamine (25.5\%). In a hypotensive hemodynamic situation, norepinephrine was combined with PDE-III blockers (63.8\%), inhaled prostanoids (38.3\%), or dobutamine (34.0\%). Only $19.0 \%$ of respondents reported to use adrenaline combined with nitroglycerine as the first-line treatment in this situation.

\section{Discussion}

The present study aimed at assessing the prevalence of preoperative RVD and PAH, the measures used to diagnose these risk factors, as well as treatment practice in adult cardiac surgery in Germany by a postal survey.

The results clearly show that these entities remain frequently undetected preoperatively. This contradicts the fact that preoperative RVD and PAH are recognized as clinically relevant problems in the care of cardiac surgical patients and by the observation that most centers have even established concepts for the pre- and perioperative treatment and monitoring of RVD and PAH. Our results provide evidence that that more than $50 \%$ of all CABG cases do not undergo routine preoperative echocardiography for evaluation of right ventricular performance and PAP. Comparably, in patients scheduled for valve surgery (in which a preoperative echocardiography is mandatory to determine the extent of valvular disease), right ventricular catheterization is also only rarely performed despite increasingly recognized limitations of echocardiography for the precise determination of PAPs in patients with $\mathrm{PAH}^{14}$ and severe tricuspid regurgitation. ${ }^{15}$ Interestingly, in centers that had data on the prevalence of these comorbidities, $19.5 \%$ of patients presented right heart dysfunction and $10 \%$ presented PAH, numbers clearly higher than those contemporarily reported. ${ }^{1,11}$

\section{[\%]}

100

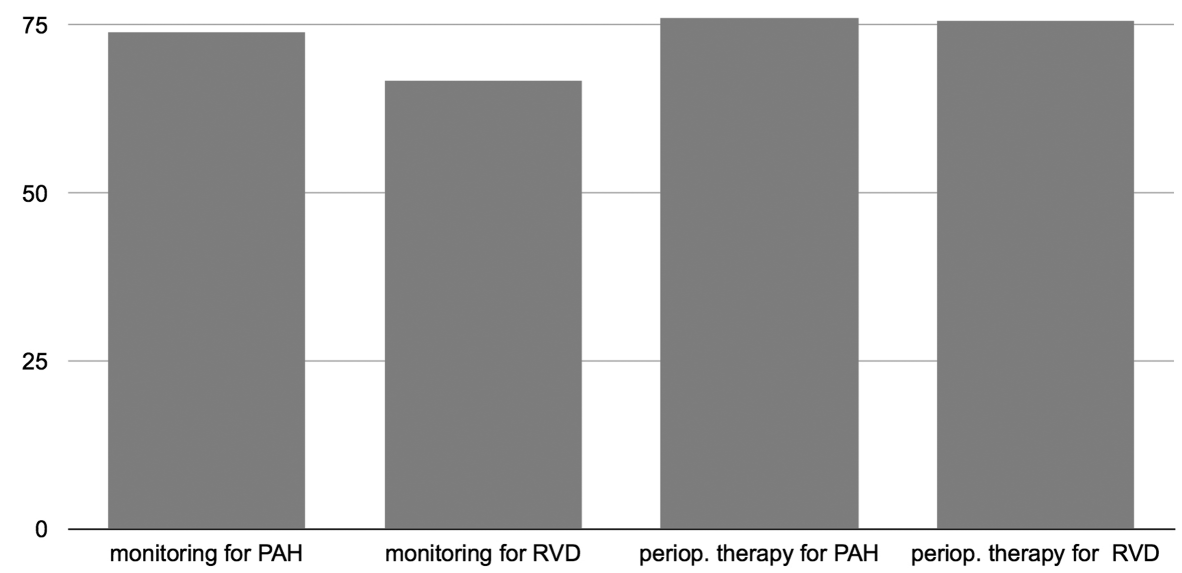

Fig. 4 Availability of standard procedures for monitoring and treatment of right ventricular dysfunction (RVD) or pulmonary arterial hypertension (PAH) in German heart centers. 
These findings may have relevant implications:

1. First of all, the fact that more than $50 \%$ of CABG patients do not have a preoperative echocardiographic or right-heart catheter examination highly questions the reliability of preoperative risk adjustments with any EuroSCORE version because pulmonary arterial pressure is one of the variables needed to calculate these scores. ${ }^{12,16,17}$

2. An increasing number of publications clearly show that preoperative right heart dysfunction is associated with a poor prognosis in cardiac surgery. ${ }^{4,11}$ Further, based on the results of this survey, clinicians either routinely initiate or are willing to initiate specific therapeutic measures (mostly PDE-V inhibitors and/or inhaled pulmonary arterial vasodilators) in patients with known RVD and/or PAH. Consequently, this lack of information may be invariably associated with inappropriate preoperative treatment in this group of high-risk cardiac surgical patients.

The reasons for this inappropriate preoperative diagnostic workup are clearly speculative.

A. First, more than half of the preoperative echocardiographic examinations were performed by an external cardiologist, not necessarily aware of the implications of RVD for the perioperative course. Thus, one may speculate that these examinations were more focused on left than on right ventricular function, especially in patients scheduled for CABG. Additionally, in the light of increased financial pressure on cardiological departments, a high number of coronary artery interventions may be more important than a time-consuming preoperative evaluation of a surgical patient including right heart catheterization.

B. As a second point and as a result of the increasing economic pressure in the German health system, repeated examinations are strongly discouraged by the health care authorities and insurance companies and cannot be reimbursed. Therefore, many institutions may be reluctant to repeat preoperative examinations-even if the quality or the information brought by the available examinations is not optimal-or may simply rely on the results of levocardiography.

C. Third, it is well known that echocardiographic determination of PAP is not possible in a relevant number of patients who-if being studied invasively by right heart catheterization-are diagnosed to have PAH. Analyzing data from 29 trials, Janda and coworkers concluded that "Echocardiography is a useful and noninvasive modality for initial measurement of pulmonary pressures, but due to limitations, right heart catheterization should be used for diagnosing and monitoring pulmonary hypertension." 18 In a more recent meta-analysis, but only analyzing nine trials, Taleb and coworkers stated that "Doppler echocardiography is a useful noninvasive modality to screen for pulmonary hypertension and can reliably determine whether systolic PAP is normal, mildly elevated, or markedly elevated. However, abnormal results from Doppler echocardiography need to be confirmed by right heart catheterization." ${ }^{19}$ This sug- gests that preoperative diagnostics in patients with presumed or documented RVD and/or PAH scheduled for cardiac surgery ideally should also include right heart catheterization. Thus, the attitude to rely only on echocardiography carries the risk to underestimate the true prevalence of PAH.

D. Last, this lack of proper preoperative diagnostics may be related to a lack of established recommendation, that is, the fact that there are no guidelines suggesting a diagnostic workup of right ventricular function and the pulmonary circulation in patients undergoing cardiac surgery. And indeed, despite a recent German guideline on hemodynamic monitoring and treatment of patients after cardiac surgery recommending the use of extended hemodynamic monitoring in patients with right heart dysfunction, no suggestions are made regarding preoperative diagnostics. ${ }^{20}$ Comparably, the most recent US guideline for $\mathrm{CABG}^{21}$ only gives evidence-based recommendations on perioperative monitoring including TEE but no details or suggestions on preoperative functional cardiac diagnostics. In contrast, the most recent European guideline clearly recommends an echocardiographic examination before and after CABG with a class I, level C recommendation. ${ }^{22}$ Unfortunately, this information is detailed in a table entitled "Long-Term Lifestyle and Risk Factor Management after Myocardial Revascularization" and thus may be easily overseen.

The respondents presented a variety of echocardiographic variables they deemed relevant for the preoperative assessment of right ventricular function. Astonishingly, objective variables for the determination of right ventricular contractility such as TAPSE, fractional area change (FAC), or the right ventricular index of myocardial performance (RIMP) were rather infrequently reported and the overwhelming number of respondents primarily focused on PAP and right atrial and ventricular dimensions. A detailed guideline on echocardiographic assessment of the right ventricle has been published after the survey ${ }^{23}$ and will possibly lead to a more standardized approach for preoperative echocardiographic assessment.

Paralleling the publication of an S3 guideline on postoperative hemodynamic monitoring and treatment in patients after cardiac surgery in 2007 and its revision in 2010,24 Kastrup and colleagues performed two surveys to determine the particular practice patterns in German cardiac surgical intensive care and how clinical practice had changed after the publication of the respective guidelines. ${ }^{24,25}$ The present analysis confirms many of their findings, especially that PAC and TEE are still the preferred monitoring modalities in German heart centers. However, Kastrup's surveys did not specifically address the monitoring used in patients with right heart dysfunction and/or PAH, a fact that may explain the much higher usage of a pulmonary artery catheter and/or TEE in these patients in the present study. Remarkably, according to the results of the present survey, RVD and RHF are most frequently treated with PDE-III inhibitors and-if 
necessary to maintain adequate blood pressure-with noradrenaline. This is in clear contrast to the results presented by Kastrup and coworkers reporting that right heart failure in German cardiac surgical ICUs is predominantly treated with adrenaline. Addressing the complete perioperative period, our survey adds new information on preoperative treatments, that is, the use of oral drugs for pulmonary vasodilatation such as sildenafil or endothelin, antagonists that may be taken into account in future revisions of this guideline. ${ }^{20}$

\section{Limitations}

Several limitations apply to the present study:

1. The methodology of a postal survey, the descriptive analysis, and the fact that nearly $75 \%$ of the answers on preoperative diagnostics were estimated and not based on prospectively sampled data must of course be recognized as relevant limitations. In any case, even if the absolute numbers may be slightly different from the numbers perceived by the respondents of this survey, the main results, that is, that no information on right ventricular function and/or PAP has been collected on a relevant number of cardiac surgical patients, are still most likely correct.

2. Regarding a lack of a generally accepted definition of RVD during the survey period, any statement about the incidence of preoperative RVD may also be regarded as relatively "weak." However, the fact that almost $80 \%$ of centers cannot give precise data on the prevalence of RVD/ RVF is clearly supportive that these comorbidities are grossly underdiagnosed in patients undergoing cardiac surgery in Germany. Interestingly, in other countries (i.e., the United States), routine data on the prevalence of $\mathrm{RVD} / \mathrm{RVF}$ are also only recorded in closed registries such as the STS (Society of Thoracic Surgeons) database and are not publicly available, suggesting that this lack of knowledge on the prevalence of pre- and perioperative RVD in cardiac surgical patients may not be restricted to Germany.

Keeping these limitations in mind, the results of the present survey provide evidence that in a relevant proportion of patients undergoing cardiac surgery in Germany, no preoperative data on right ventricular function and/or pulmonary arterial pressure are available. Additionally, most centers do not have precise data on the incidence of postoperative right heart dysfunction and/or PAH. This may lead to underestimation of perioperative risk and inappropriate management of this high-risk population, despite a high level of clinical awareness for these comorbidities and willingness to adequately react therapeutically.

In conclusion, right heart dysfunction, right heart failure, and PAH appear to represent inadequately diagnosed clinical problems in German cardiac surgery, although the importance of these problems is well acknowledged and accepted. There is an urgent need for prospective studies analyzing the magnitude of this problem and the consequences on outcome as well as precise recommendations on preoperative diagnostics in patients scheduled for cardiac surgery.
Funding

This work was supported by institutional grants of the Department of Anesthesiology and Intensive Care Medicine, University of Lübeck and the Department of Anesthesiology, Heart and Diabetic Center NRW, Ruhr University of Bochum, Bad Oeynhausen, Germany. No external funding source has been involved.

Conflict of interest

None declared.

\section{Authors' Contributions}

Designed the study (1), participated in the design of the questionnaire (2), arranged the survey process (3), analyzed the data (4), drafted the manuscript (5), revised the manuscript for important intellectual content (6), and approved the final manuscript (7).

Matthias Heringlake 1, 2, 4, 5, 6

Julika Schön 1, 2, 4, 5, 6

Teresa Pliet 1, 2, 4, 5, 6

Nils Haake 1, 2, 6, 7

Alexander Reinecke 2, 6, 7

Marit Habicher 2, 6, 7

Michael Sander 1, 2, 6, 7

Andreas Markewitz 1, 3, 5, 7

Daniel A. Reuter 1, 2, 6, 7

Heinrich Volker Groesdonk 1, 2, 6, 7

Georg Trummer 1, 2, 6, 7

Kevin Pilarzyk 1, 2, 6, 7

Michael von der Brelie 1, 2, 6, 7

Berthold Bein 1, 2, 6, 7

Uwe Schirmer 3, 5, 7

Note

This abstract was presented at the annual meeting of the European Association of Cardiothoracic Anesthetists (EACTA) in Barcelona, Spain on June 7th, 2013.

\section{References}

1 Haddad F, Couture P, Tousignant C, Denault AY. The right ventricle in cardiac surgery, a perioperative perspective: II. Pathophysiology, clinical importance, and management. Anesth Analg 2009; 108(2):422-433

2 Maslow AD, Regan MM, Panzica P, Heindel S, Mashikian J, Comunale ME. Precardiopulmonary bypass right ventricular function is associated with poor outcome after coronary artery bypass grafting in patients with severe left ventricular systolic dysfunction. Anesth Analg 2002;95(6):1507-1518

3 Boeken U, Feindt P, Litmathe J, Kurt M, Gams E. Intraaortic balloon pumping in patients with right ventricular insufficiency after cardiac surgery: parameters to predict failure of IABP support. Thorac Cardiovasc Surg 2009;57(6):324-328

4 Haddad F, Denault AY, Couture P, et al. Right ventricular myocardial performance index predicts perioperative mortality or circulatory failure in high-risk valvular surgery. J Am Soc Echocardiogr 2007;20(9):1065-1072 
5 Yadav H, Unsworth B, Fontana M, et al. Selective right ventricular impairment following coronary artery bypass graft surgery. Eur J Cardiothorac Surg 2010;37(2):393-398

6 Schosser R, Forst H, Racenberg J, Messmer K. Open chest and open pericardium affect the distribution of myocardial blood flow in the right ventricle. Basic Res Cardiol 1990;85(5):508-518

7 Unsworth B, Casula RP, Kyriacou AA, et al. The right ventricular annular velocity reduction caused by coronary artery bypass graft surgery occurs at the moment of pericardial incision. Am Heart J 2010;159(2):314-322

8 de Groote P, Millaire A, Foucher-Hossein C, et al. Right ventricular ejection fraction is an independent predictor of survival in patients with moderate heart failure. J Am Coll Cardiol 1998;32(4):948-954

9 Murninkas D, Alba AC, Delgado D, et al. Right ventricular function and prognosis in stable heart failure patients. J Card Fail 2014; 20(5):343-349

10 Mohammed SF, Hussain I, AbouEzzeddine OF, et al. Right ventricular function in heart failure with preserved ejection fraction: a community-based study. Circulation 2014;130(25): 2310-2320

11 Garatti A, Castelvecchio S, Di Mauro M, Bandera F, Guazzi M, Menicanti L. Impact of right ventricular dysfunction on the outcome of heart failure patients undergoing surgical ventricular reconstruction. Eur J Cardiothorac Surg 2015;47(2):333-340, discussion 340

12 Nashef SA, Roques F, Michel P, Gauducheau E, Lemeshow S, Salamon R. European system for cardiac operative risk evaluation (EuroSCORE). Eur J Cardiothorac Surg 1999;16(1):9-13

13 Strumpher J, Jacobsohn E. Pulmonary hypertension and right ventricular dysfunction: physiology and perioperative management. J Cardiothorac Vasc Anesth 2011;25(4):687-704

14 Fisher MR, Forfia PR, Chamera E, et al. Accuracy of Doppler echocardiography in the hemodynamic assessment of pulmonary hypertension. Am J Respir Crit Care Med 2009;179(7):615-621

15 Özpelit E, Akdeniz B, Özpelit EM, et al. Impact of severe tricuspid regurgitation on accuracy of echocardiographic pulmonary artery systolic pressure estimation. Echocardiography 2015;32(10): 1483-1490

16 Roques F, Michel P, Goldstone AR, Nashef SA. The logistic EuroSCORE. Eur Heart J 2003;24(9):881-882
17 Nashef SA, Roques F, Sharples LD, et al. EuroSCORE II. Eur J Cardiothorac Surg 2012;41(4):734-744, discussion 744-745

18 Janda S, Shahidi N, Gin K, Swiston J. Diagnostic accuracy of echocardiography for pulmonary hypertension: a systematic review and meta-analysis. Heart 2011;97(8):612-622

19 Taleb M, Khuder S, Tinkel J, Khouri SJ. The diagnostic accuracy of Doppler echocardiography in assessment of pulmonary artery systolic pressure: a meta-analysis. Echocardiography 2013; 30(3):258-265

20 Carl M, Alms A, Braun J, et al. S3 guidelines for intensive care in cardiac surgery patients: hemodynamic monitoring and cardiocirculary system. Ger Med Sci 2010;8:Doc12

21 Hillis LD, Smith PK, Anderson JL, et al. 2011 ACCF/AHA Guideline for Coronary Artery Bypass Graft Surgery: a report of the American College of Cardiology Foundation/American Heart Association Task Force on Practice Guidelines. Circulation 2011;124(23): e652-e735

22 Wijns W, Kolh P, Danchin N, et al; Task Force on Myocardial Revascularization of the European Society of Cardiology (ESC) and the European Association for Cardio-Thoracic Surgery (EACTS); European Association for Percutaneous Cardiovascular Interventions (EAPCI). Guidelines on myocardial revascularization. Eur Heart J 2010;31(20):2501-2555

23 Rudski LG, Lai WW, Afilalo J, et al. Guidelines for the echocardiographic assessment of the right heart in adults: a report from the American Society of Echocardiography endorsed by the European Association of Echocardiography, a registered branch of the European Society of Cardiology, and the Canadian Society of Echocardiography. J Am Soc Echocardiogr 2010;23(7):685-713, quiz 786-788

24 Kastrup M, Markewitz A, Spies C, et al. Current practice of hemodynamic monitoring and vasopressor and inotropic therapy in postoperative cardiac surgery patients in Germany: results from a postal survey. Acta Anaesthesiol Scand 2007;51(3):347-358

25 Kastrup M, Carl M, Spies C, Sander M, Markewitz A, Schirmer $\mathrm{U}$. Clinical impact of the publication of S3 guidelines for intensive care in cardiac surgery patients in Germany: results from a postal survey. Acta Anaesthesiol Scand 2013;57(2): 206-213 